\title{
An Optimal Adaptive Flight Control System
}

\author{
Mojtaba Vahedi, Ali Akbarzadeh Kalat, and Mohammad Hadad Zarif
}

\begin{abstract}
In This paper, an optimal self tuning regulator (STR) structure is applied to a nonlinear flight system. In control structure, a modified GA algorithm for obtaining a suitable observer polynomial is proposed which optimizes the controller performance. The proposed method has two major advantages; first of all, it is independent of system degree or system complexity and secondly, in this method some of unknown STR method parameters such as observer polynomial are discarded. The designed controller is applied to a F-18 nonlinear model. Simulation results are presented which show that in the closed-loop system asymptotic trajectory control is accomplished. Also computer simulations are carried out for showing the performance of the designed controller against common STR controller.
\end{abstract}

Index Terms-Self tuning regulator, genetic algorithm, flight control.

\section{INTRODUCTION}

High performance aircraft control poses a unique challenge to a control designer due to stringent performance requirements, system complexities and large variations in dynamics over the complete flight envelope. Additionally, for an extremely maneuverable aircraft, meeting performance specifications can be particularly challenging because of difficulties involved in modeling or estimating the nonlinearities associated with such an aircraft.

The research done in the area of flight control has been vigorous these past years and there are various control design techniques being used by the industry to design aircraft controllers. These techniques include Proportional-Integral (PI) control, Optimal Linear Quadratic Regulator / Linear Quadratic Gaussian (LQR/LQG) control, $\mu$ synthesis robust control, $H_{\infty}$ control, dynamic inversion, neural network, adaptive control and Linear Parameter Varying (LPV) control [1].

This clearly indicates that the industry has embraced advanced control techniques that are now the standard for designing flight control laws for advanced high performance aircraft.The above mentioned techniques have been the subject of research these past few years and have provided vital contributions to the field of flight control performance.

For instance, the Herbst like maneuver performed on a nonlinear fighter aircraft demonstrates the robustness of a radial basis neural network based controller [2]. On the Other hand, a Model Reference Adaptive Control (MRAC) based

Manuscript received August 9, 2012; revised November 21, 2012.

M. Vahedi is with the Electrical and Computer Department, Islamic Azad University, Shahrood branch. Shahrood, Iran (e-mail: vahedi.mojtaba@yahoo.com).

A. Akbarzadeh Kalat and M. Hadad Zarif are with the Electrical and robotic Engineering Department, shahrood university of technology, Shahrood, Iran (e-mail: aliakkalat@yahoo.com; mhzarif@shahroodut.ac.ir). adaptive controller is designed for aircraft pitch rate tracking using multiple fuzzy reference model technique [2] and neural network[3]. An alternate approach augments multiple modern control tools such as neural network, fuzzy logic, $\mu$ synthesis and $H_{\infty}$ control to perform Herbst like complex maneuver [4]. A combination of stochastic robustness and dynamic inversion is also proposed for high performance aircraft control application in research literature [5]. Sliding Mode Control of Pitch Rate for an F-16 is proposed in [6]. An adaptive controller design method based on back-stepping neural network is proposed for reconfigurable flight control systems in the presence of variations in aerodynamic coefficients or control effectiveness deficiencies caused by control surface damages [7].Adaptive control schemes are used to overcome difficulties connected with the ignorance of system structure, where the parameters of the plant are not known exactly or slowly time varying [8], [9], [10]. For the control of plants different approaches have been adopted to design the adaptive controllers.

This paper is based on an adaptive control scheme known as Self Tuning Regulator (STR). A linearized model of an aircraft operated at a specific flight condition is used to design a controller. Linear controller designed using this method provides good performance near the design point. In order to guarantee the desired performance over a wide range of flight envelope, the gain scheduling technique is used on multiple linear controllers designed for different operating points [11], [12].

In this paper genetic algorithm has been used to optimize STR unknown parameters such as observer polynomial. [8] represented a method for accomplishing the selection of observer but, there is no guarantee that this is practical in flight or complicated systems. In order to soften this problem, a modified GA algorithm for obtaining a suitable observer polynomial is proposed, which improve the controller performances. The proposed method has two major advantages in compare with other methods; first of all, the proposed method is independent of system degree or system complexity. Secondly, in this method some of unknown STR parameters are discarded and designers do not need to estimate them.

\section{Self Tuning Regulator}

\section{A. Self-Tuning Regulator}

Adaptive control methods were developed as an attempt to overcome difficulties connected with the ignorance of system structure and critical parameter values as well as changing control regimes. Different approaches have been adopted to design the adaptive controllers. The self-tuning regulator attempts to automate the tasks involved in the adaptive 
control scheme namely modeling, design of a control law, implementation, and validation. This is illustrated in Fig. 1. The parameters of the process model are estimated online by a recursive estimator such as recursive least square (RLS). The block labeled "estimation" gives an estimate of the process parameters. The block labeled "controller" is an implementation of the controller whose parameters are obtained from the "controller design".

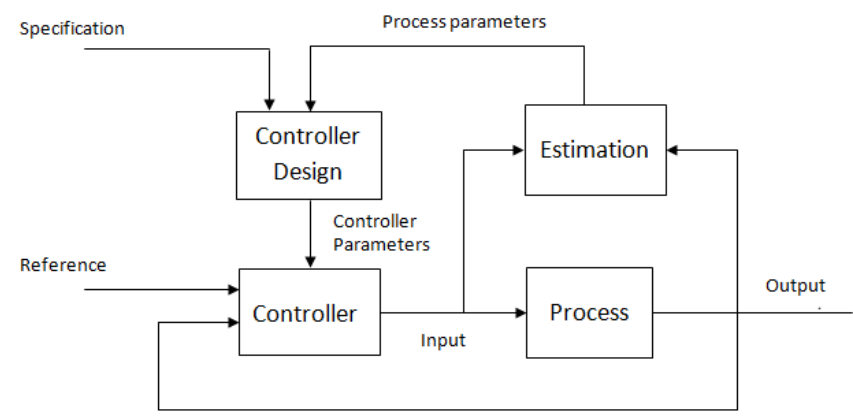

Fig. 1. Block diagram of a self-tuning regulator

It is assumed that the process is described by the SISO system

$$
A y(t)=B(u(t)+v(t))
$$

where $\mathrm{y}$ is the output, $\mathrm{u}$ is the input of the process and $\mathrm{v}$ is a disturbances. A and B are denote polynomials in either the differential operator $P=d / d t$ or the forward shift operator q Also it is assumed that A and B is relatively prime, and $\mathrm{A}$ is monic.

A general linear controller can be described by

$$
R u(t)=T u_{c}(t)-S y(t)
$$

where $\mathrm{R}, \mathrm{S}$, and $\mathrm{T}$ are polynomials. This control law represents a negative feedback with a transfer operator $S / R$ and a feed forward with the transfer operator T/R. it thus has two degrees of freedom. A block diagram of the closed-loop system is shown in Fig. 2.

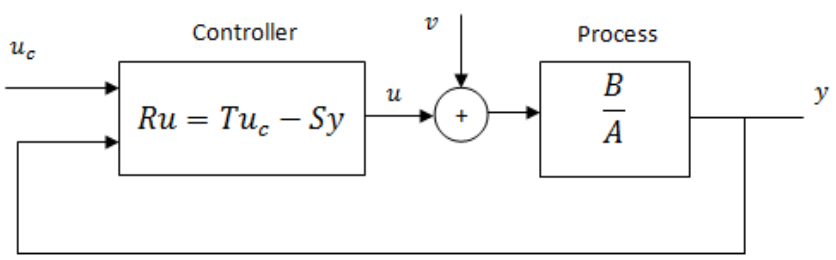

Fig. 2. A general linear controller with two degrees of freedom

Elimination of $u$ between (1) and (2) gives the following equations for the closed-loop system

$$
\begin{gathered}
y(t)=\frac{B T}{A R+B S} u_{c}(t)+\frac{B R}{A R+B S} v(t) \\
u(t)=\frac{A T}{A R+B S} u_{c}(t)-\frac{B S}{A R+B S} v(t)
\end{gathered}
$$

The closed-loop characteristic polynomial is thus

$$
A R+B S=A_{c}
$$

The key idea of the design method is to specify the desired closed-loop characteristic polynomial $A_{C}$. The polynomials $\mathrm{R}$ and $\mathrm{S}$ can then be solved from (4). Equation (4) which plays a fundamental role in algebra is called the Diophantine equation. This equation always has solutions if the polynomials A and B do not have common factors.

The Diophantine equation determines only the polynomials $\mathrm{R}$ and $\mathrm{S}$. other conditions must be introduced to also determine the polynomial $\mathrm{T}$ in the controller (2). To do this, we will require that the response from the command signal $u_{c}$ to the output be described by the dynamics

$$
A_{m} y_{m}(t)=B_{m} u_{c}(t)
$$

It then follows from (3) that the following condition must hold. [8]

$$
\frac{B T}{A R+B S}=\frac{B T}{A_{c}}=\frac{B_{m} A_{o}}{A_{m} A_{o}}
$$

This model-following condition says that the response of the closed-loop system to command signals is as specified by the model (5). In this equation $A_{o}$ is monic and called observer polynomial [8]. The observer polynomial is canceled in the transfer function from command signal to process output.

\section{B. Causality Conditions}

For obtain a causal controller with lowest degree, we must impose the conditions [8]

$$
\begin{gathered}
\operatorname{deg} S \leq \operatorname{deg} R \\
\operatorname{deg} T \leq \operatorname{deg} R \\
\operatorname{deg} A_{c} \leq 2 \operatorname{deg} A-1 \\
\operatorname{deg} A_{o} \leq 2 \operatorname{deg} A-\operatorname{deg} A_{m}-1 \\
\operatorname{deg} A-\operatorname{deg} B \leq \operatorname{deg} A_{m}-\operatorname{deg} B_{m}
\end{gathered}
$$

\section{Indirect Self-Tuning Regulator}

Combining the recursive least squares (RLS) estimator with the minimum-degree pole placement method (MDPP) for controller design, we obtain the following self tuning regulator. This method's Algorithm is:

1) Data: given specification in the form of a desired closed-loop pulse transfer operator $B_{m} / A_{m}$ and a desired observer polynomial $A_{o}$.

2) Step1: estimate the coefficient of the polynomials A and $\mathrm{B}$ in (1) using the recursive least-squares method.

3) Step2: solving the Diophantine equation where $A$ and $B$ are the estimates obtained in step 1 . The polynomials R, $\mathrm{S}$ and $\mathrm{T}$ of the control law then obtained.

4) Step3: calculate the control variable, that is:

$$
R u(t)=T u_{c}(t)-S y(t)
$$

5) Repeat steps 1,2 and 3 at each sampling period. 


\section{MATERIAL AND METHODS}

The self tuning regulator or STR is a linear controller. First of all, F-18 nonlinear model is linearized by Jacobian method over accumulation point and then, obtained linear model is used for controller design.

The states used to define longitudinal channel are velocity $(v)$, angle of attack $(\alpha)$, pitch angle $(\theta)$ and pitch rate $(q)$. In this paper, pitch angle is considered as output and elevator deflection $(\delta e)$ is its corresponding control input. The purpose is to design an adaptive controller with fast and accurate command tracking. For flight condition of $10000 \mathrm{ft}$ altitude and $v=500 \mathrm{ft} / \mathrm{sec}$, the respective transfer function for pitch angle is:

$$
G(s)=\frac{0.001133 S+0.01394}{S^{4}+1.004 S^{3}+2.299 S^{2}+0.02555 S+0.02157}
$$

Also the selected reference model is:

$$
G m(s)=\frac{4 S^{2}+14 S+10}{S^{4}+6 S^{3}+15 S^{2}+20 S+10}
$$

In Self-Tuning-Regulators the observer polynomial is highly important and should be chosen properly. In the mean time, observer polynomial's coefficient cannot be obtained by classical methods therefore, in this paper GA is used for choosing the best observer polynomial from the total set of acceptable polynomial (which is satisfied the Causality conditions in last section). The parameters used in GA are as follows:

1) Variables are the coefficients of observer polynomial.

2) Number of variables are 3 (because of causality conditions).

3) All the variable spaces are the same and are equal to [0 $10]$.

4) Number of bits in each variable is selected as 20 so that the total length of each population is 60 .

5) The number of initial populations is assumed to be 256 . In the mean time, the populations that can not satisfy causality conditions are regenerated.

6) The number of cross points is assumed to be 9 according to Alavi gharahbagh [13].

7) According to Alavi gharahbagh [13] the probability of mutation or P Mute is assumed to be 0.02 .

8) Answer accuracy is a rational factor for breaking computation process.

$$
\text { Answer Accuracy }=\frac{\mid \text { Thebest answer }- \text { the worst answer } \mid}{\text { Thebest answer }} \times 100
$$

And this value is assumed $0.02 \%$.

1) The cost function is the summation of output maximum settling time and output maximum overshoot that should be minimized.

By using GA with above conditions, the best observer can be determined as follows:

$$
\text { Ao }(s)=S^{3}+0.1697 \mathrm{~S}^{2}+0.1256 \mathrm{~S}+0.0027
$$

Fig. 3 shows the convergence of plant parameters in proposed STR method.
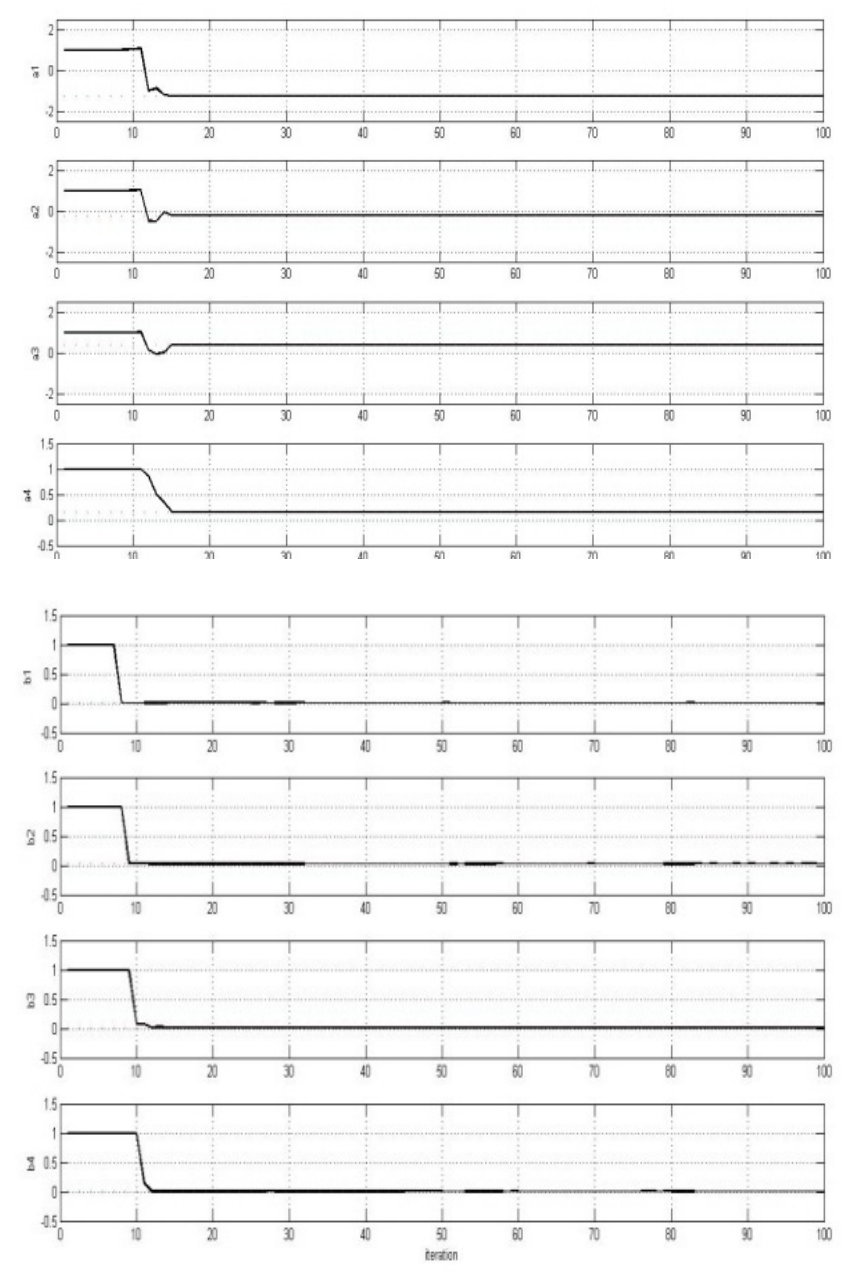

Fig. 3. Convergence of plant parameters

\section{Simulation Result}

The designed adaptive controller is simulated based on following cases:

\section{A. Situations Out of Accumulation Point}

In this Simulation, a nonlinear model of a flight object with 6 degrees of freedom is considered. This system outputs should be tracked pitch angle commands. System is simulated over a wide range of commands around accumulation point. The close-loop system response to constant input $(\theta=10)$ is illustrated in Fig. 4. This picture emphasis that the proposed system tracks input commands very good. For illustrating designed controller reliability, a time variant input for pitch angles is applied to system. The result and it's control efforts is shown in Fig. 5. If the system designed poorly, the amplitude of control signals would be very large and does not work properly for actuators, but in proposed system these values are in the acceptable range.

\section{B. Comparison between Designed STR and Common Self} -Tuning Regulator

In this section, proposed controller is compared with common STR controller. In the design of common STR controller an acceptable observer polynomial according to the causality conditions is selected [8]. This polynomial is:

$$
A o(s)=S^{3}+0.1 \mathrm{~S}^{2}+0.2 \mathrm{~S}+0.3
$$


Based on the algorithm noted in [8], the common STR has designed. From the comparison results, it is obvious that designed controller based on GA is much better than common STR which is shown in Fig. 6.

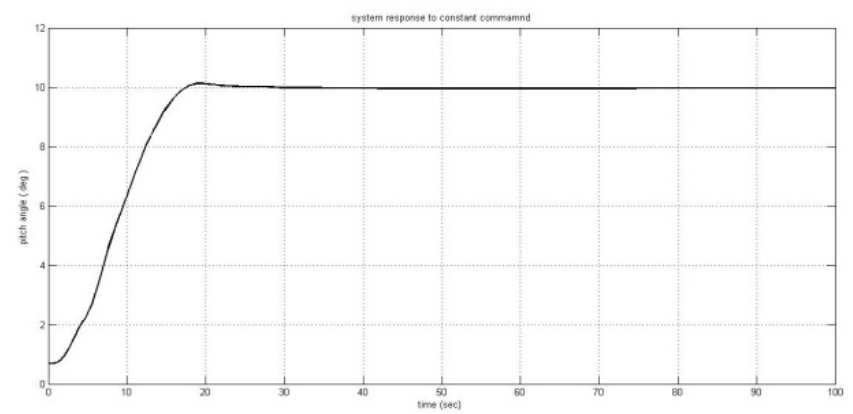

Fig. 4. Nonlinear close-loop system response for $\theta=10$.

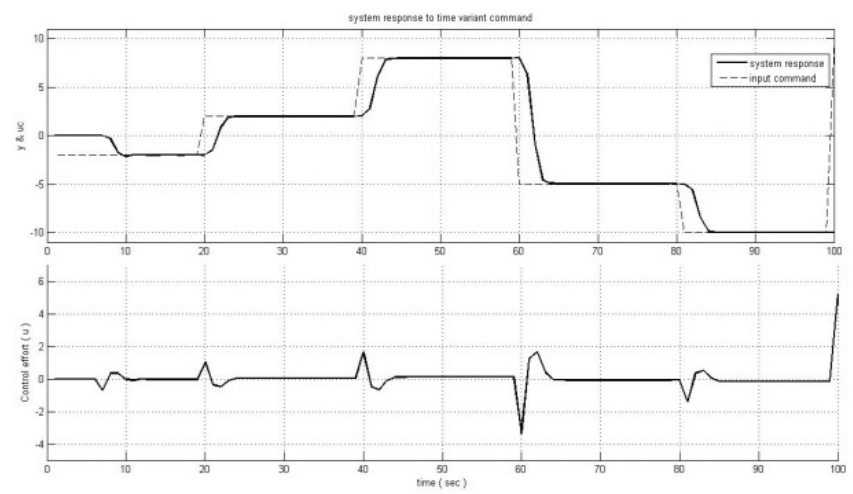

Fig. 5. Nonlinear system response for pitch command and its control effort

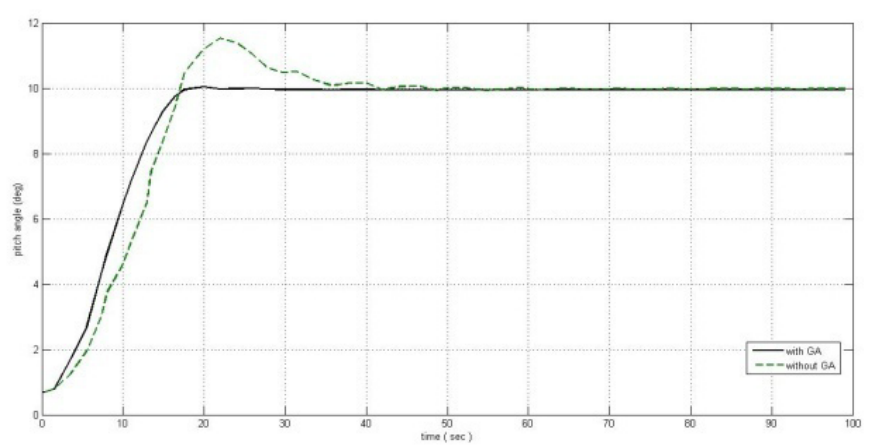

Fig. 6. Designed controller based on GA responses in compare with common STR controller

\section{CONCLUSION}

In this paper a self tuning regulator for a flight object optimized by genetic algorithm. This controller required a suitable observer polynomial. To fulfill this requirement, GA is used. The designed controller was tested on a nonlinear 6 degrees of freedom flight model. All simulation results showed system reliability and stability in practical situations. In addition, simulation results showed that the time response of designed controller based on GA is much better than common STR controller. Moreover, the proposed controller has a good performance in a wide range of varieties over accumulation point in compare to other controllers.

\section{REFERENCES}

[1] G. J. Balas, "Flight control law design: An industry perspective," European J. of Control, vol. 9, no. 2-3, pp. 207- 226, 2003.

[2] S. Kamalasadan and A. A. G handakly, "Multiple fuzzy reference model adaptive controller design for nonlinear fighter aircraft pitch-rate tracking," IEEE Trans. of Instrumentation and Measurements, vol. 56, no. 5, pp. 1797-1808, Oct. 2007.

[3] S. Kamalasadan and A. A. G handakly, "A neural network parallel adaptive controller for dynamic systems control," IEEE Trans. of Instrumentation and Measurements, vol. 56, no. 5, pp. 1786-1796.

[4] J. Wang and W. Zhang, "Neural-fuzzy scheduling of $\mathrm{H}$ robust controllers for a high performance fighter aircraft under a Herbst-like maneuver," Int. J. of Control, vol. 72, no. 7, pp. 740-754, 1999.

[5] Q. Wang and R. F. Stengel, "Robust nonlinear flight control of a high performance aircraft," IEEE Trans. on Control Systems Technology, vol. 13 , no. 1, pp. 15-26, January 2005 .

[6] E. Promtun and S. Seshagiri, "Siding mode control of pitch-rate of an F-16 aircraft," 2009

[7] D-H. Shin and Y. Kim, "Reconfigurable flight control system design using adaptive neural networks," IEEE Trans. on Control Systems Tech., vol. 12, no. 1, pp. 87-100, Jan. 2004.

[8] K. J. Astrom, and B. Wittenmark, Adaptive Control, 2nded, Addison-Wesley Publishing Company Inc., New York, NY, 1995.

[9] K. J. Astrom and B. Wittenmark, "Self-tuning controller based on pole-zero placement," IEEE Proc. D, 120:120-130, 1980.

[10] T. Yahagi and J. Lu, "On self-tuning control of non-minimum phase discrete time systems using approximate inverse systems," Journal of Dynamic Systems, Measurement, and Control, 115: 12-18, 1993.

[11] S. A. Snell, D. F. Enns, and W. L. Garrard, "Nonlinear control of a super maneuverable aircraft," in Proc. AIAA GN\&C Conf., pp. 519-531. 1989.

[12] S-L. Wu, P-C. Chen, C-H. Hsu, and K-Y. Chang, "Gain-scheduled control of PVTOL aircraft dynamics with parameter-dependent disturbance," J. of the Franklin Institute, vol. 345, no. 8, pp. 906-925, Nov.2008.

[13] A. G. Abdorreza and A. Vahid. "A Novel Accurate Genetic Algorithm for Multivariable Systems," World Applied Sciences Journal vol. 5, no. 2, pp. 137-142, 2008. 